\title{
Self Adaptable Deployment for Heterogeneous Wireless Sensor Network
}

\author{
Umesh M. Kulkarni ${ }^{1}$, Harish H. Kenchannavar ${ }^{2}$, Umakant P. Kulkarni ${ }^{3}$ \\ Dept. of CSE, KLS's Gogte Institute of Technology, Belagavi, India. VTU, Belagavi ${ }^{1}$ \\ Dept. of CSE, KLS's Gogte Institute of Technology, Belagavi, India. VTU, Belagavi ${ }^{2}$ \\ Prof., Dept. of CSE, SDM college of Engineering and Technology, Dharwad, India. VTU, Belagavi ${ }^{3}$
}

\begin{abstract}
Wireless Sensor Networks (WSN) is becoming a crucial component of most of the fields of engineering. Heterogeneous WSN (HWSN) is characterized by wireless sensor nodes having link (communication), computation or energy heterogeneity for a specific application. WSN applications are constrained by the availability of power hence; conserving energy in a sensor network becomes a major challenge. Literature survey shows that node deployments can have good impact on energy conservation. Works show that self-adaptable nodes can significantly save energy as compared to other types of deployment. This work uses the concept of self-adaptation of nodes to conserve energy in a HWSN. A deployment strategy driven by some dynamic decision making capability can boost the overall performance of a WSN. The work presents an analysis of three types of deployments: like keeping all nodes fixed, all node moving and high energy nodes moving with respect to throughput, delay and energy consumption. Experimental results show that self-adaptable dynamic deployment gives $10 \%$ better throughput and $6 \%$ better energy conservation than static deployment strategies.
\end{abstract}

Keywords-Wireless sensor network (WSN); deployment strategy; self-adaptable

\section{INTRODUCTION}

Wireless Sensor Networks (WSN's) consist of small nodes with sensing, computation and wireless communication capabilities. Recent advances in electronics and wireless communication technologies have enabled the development of large-scale WSN's, which consist of much low power, lowcost and small-size sensor nodes. The literature review presents sufficient number of methods for best utilization of existing resources. Heterogeneous WSN (HWSN) are characterized by wireless sensor nodes having link (communication), computation or energy heterogeneity for a specific application [14]. Literature shows that HWSN are going to take over homogeneous WSN [15] in the days to come. However, the heterogeneity of the nodes is not given much importance as compared to homogeneous nodes in WSN. Using any of the heterogeneity features can bring evolution in the field of WSN research. Node Deployment is one of the methods of resource utilization. Deployment of nodes means placing of nodes in an area for sensing of information for specific application. An efficient sensor node deployment or placement strategy can assure efficient resource utilization, network lifetime maximization, less end to end delay and energy utilization as well. Broadly, the deployment strategies in WSN are classified as static deployment and dynamic deployment [4][16]. Further, the static node deployments are classified as deterministic and random deployment. In a deterministic static deployment strategy, the nodes are deployed in known locations. Whereas, in random static deployments the nodes are deployed at any random locations and once deployed their location become static. This work considers the random static and random dynamic deployments with movements and without movements. Fig. 1 shows the deployment classification.

Designing an efficient sensor node deployment technique using available resources is a basic task in any of the WSN's applications. The performance of such WSN can be measured using different parameters like energy conservation, delay and throughput. Fig. 2 shows a generic model considered for the work.

Fig. 2 show the three major components in a generic node deployment namely:

- Sensing area/ point: The place for which the sensing needs to be performed.

- WSN Nodes: The nodes with sensing, computing and communication capability.

Base station: The fixed node with more capability than other nodes. All other nodes send the messages to this node.

This paper is structured into six sections starting from the introduction to results and conclusion. The introduction sections present the idea of WSN and the different deployment strategies. The literature survey section presents the current work and motivation for this paper. The third and important section describes the system model under the consideration, basic terminologies needed for understanding the working of proposed algorithm. This section also provides the simple mathematical model for proposed algorithm. The fourth and fifth section provides the details of parameters considered for experimentation and short information about the simulation tool NS2. The sixth chapter illustrates the results and graphs along with justification of the graphs. Finally the paper concludes by presenting the applicability of self-adaptable logic for HWSN. 


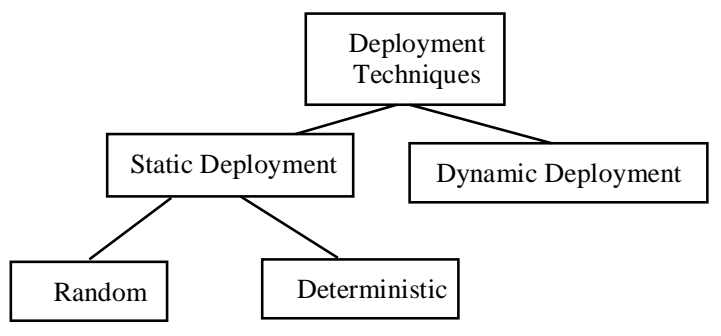

Fig. 1. Classification of Deployment Techniques.

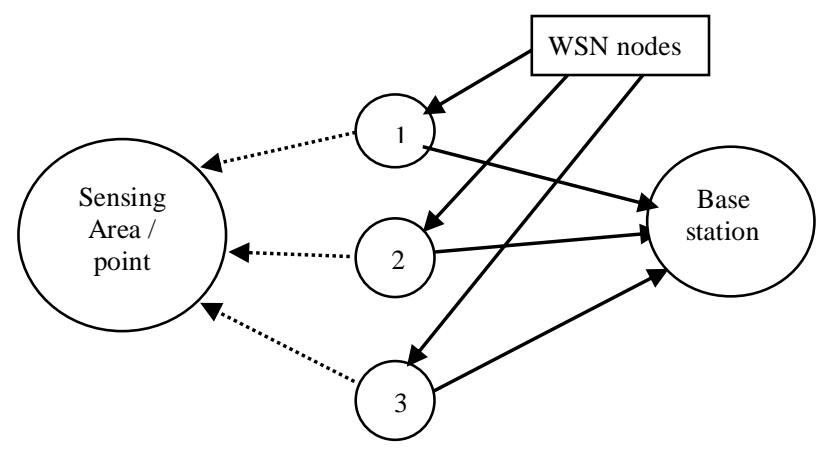

Fig. 2. Generic Node Deployment.

\section{LITERATURE SURVEY}

In [1] [2], the importance of deployment strategy is been presented. It is been clearly mentioned that the performance of a deployment strategy is dependent on coverage, network connectivity and lifetime. Even mathematical relations are been found for maximum coverage area of a node in a WSN. The correct arrangement or best topology can have better impact on the working of a WSN. The research works carried out even propose a potential based approach for the purpose of efficient deployment or arrangement of nodes. In [3], Kconnected greedy algorithm was used in the work to better deploy the nodes. This gives a hint about the use of neural networks used for decision making in WSN [3].

Energy conservation model can change according to the types of applications. The threshold distance can be related to the energy directly. Giving a hint about relation between the distance and energy. A battery aware energy efficient transmission approach was presented where the nodes work with the awareness of the energy levels with them [4].

In [5], the research work demonstrates that the energy efficiency can be achieved with respect to clustering, deployment of nodes with some modification in them. The work considers the residual energy and node density as the parameter for clustering. The paper also shows that different levels of energy of different nodes can be best utilized. In [6] [7], several other energy conservation methods like energy aware deployment strategies for video transmission. The work confirms that the energy consumption is directly proportional to the distance of nodes from base station and movement of nodes in the network. Sometimes the packet size also matters for energy conservation.

A swarm intelligence algorithm with artificial ants can increase the self-configuring capability for nodes. This work makes use of the ant colony based algorithm to give the nodes a capacity of self-organizing [8]. New algorithms can be developed at MAC layer and network layer for energy efficiency [9]. The work show that base station control for sensor network functioning can significantly save the energy. This approach even proposes a sleep scheduling adaptive algorithm that works on concept of source node and root node identification and communication between them.

According to [10] artificial intelligence and machine learning can be used for implementing some basic concepts of wireless networks. The work also discusses about using artificial neural network(ANN) for implementing some of the concepts in WSN. Especially self-organizing map (SOM) technique can be used in WSN's for clustering and grouping some of the nodes based on some criteria. The research gives direction for using concept of arrangement of nodes and some decision making capacity to it such that overall energy consumption can be reduced. Deployment, coverage and energy consumption are inter-linked with each other, as one changes the other will also change. The work even proves that the duration for which the network will be on is directly dependant on the number of active nodes in the network. That is, the work distributes the selected nodes and others are allowed to become idle. The idea behind doing this is to have better and extended sensing effect. The work even suggests that pattern based deployment and random deployment can help in boosting the energy conservation [11]. According to [12], the square grid coverage for WSN is sometimes an NPcomplete problem. According to [13], Coverage can be increased using the mobility of nodes in sensor networks. Different type of deployments and different energy levels of nodes can be considered for the study of energy conservation.

Heterogeneous nodes in a WSN can be added advantage for extending the network lifetime of sensor network[14]. The heterogeneity in the nodes comes in three ways namely link, computational and energy[14]. Any techniques in WSN that considers any of the heterogeneity factor can perform well in extending the lifetime of network [14][15]. According to [17], The lifetime can also be increased by introducing some highenergy heterogeneous sensors in the deployed network. These deployed nodes are also called as rely nodes.

\section{A. Summary}

It is found from the literature that better sensing effect and energy conservation are the major issues to be considered for hardware or software design of HWSN. Deployment of nodes will play an important role in identifying the amount of energy needed for communication. Energy used in computation, communication and distances between the communicating sensors play an important role in extending the network lifetime. Deployment of nodes with respect to the base station and the sensing area can play a vital role in extending the lifetime of network. Self adaptable node algorithms that address the energy conservation issue with better sensing effect need to be designed. Hence, a novel approach that addresses this research issue is needed.

\section{SySTEM MODEL}

This work mainly focuses on studying the effect of change of position of the nodes in the deployment area. We are considering the three scenarios as shown in Fig. 3 to 5. 


\section{A. Models of Deployment}

In this work, we are considering three models. In each of these models, the sensing area considered is shown in Fig. 3. The figure shows how the sensing area is divided into different sections Area 1 to Area 4 covered by sensor 1 to sensor 4 .

The deployment models considered for the work are as shown in Fig. 4 to 5 . Every model has different model for energy consumption calculations.

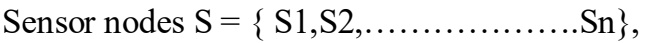

Residual energy of every node $\mathrm{E}=\{\mathrm{E} 1, \mathrm{E} 2, \ldots \ldots \mathrm{En}\}$,

Transmission energy for every node $E t=\{E t 1, E t 2, \ldots E t n\}$,

Receiving energy for every node $\mathrm{Er}=\{\operatorname{Er} 1, \mathrm{Er} 2, \ldots \ldots \mathrm{Ern}\}$,

Movement energy for every node $\mathrm{Em}=\{\mathrm{Em} 1, \mathrm{Em} 2, \ldots \mathrm{Emn}\}$.

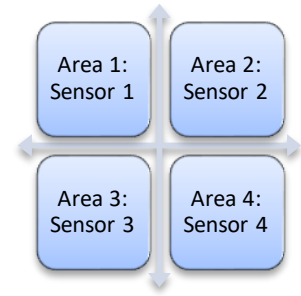

Fig. 3. Sensing Area and its Coverage by Sensors.

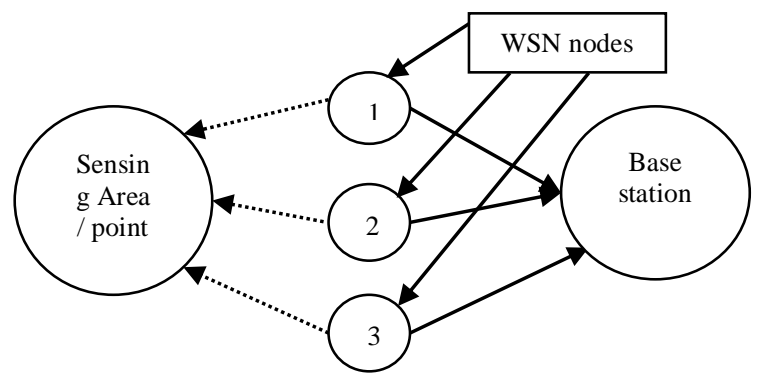

Fig. 4. Fixed Node Deployment.

At any interval of time, energy of any node $\mathrm{Sn}$ in fixed node deployment is given by:

$\mathrm{En}=\mathrm{En}-\mathrm{Etn}-\mathrm{Ern}$

At any interval of time, energy of any node Sn in All nodes moving deployment is given by:

\section{$\mathrm{En}=$ En-Etn-Ern-Emn}

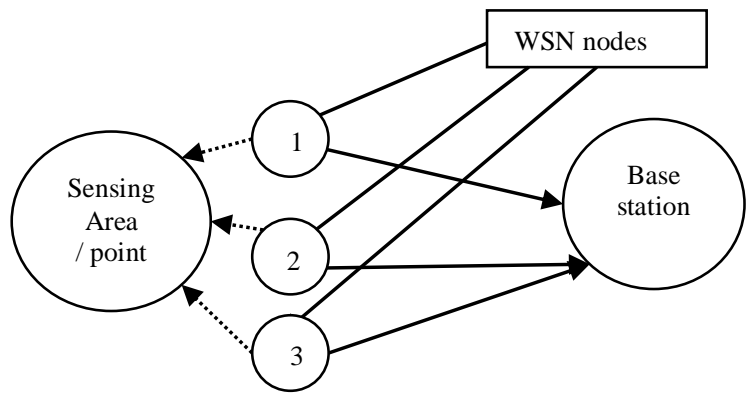

Fig. 5. Moving All Nodes.
At any interval of time energy of any sensor node $\mathrm{Sn}$ in high energy moving node deployment is given by:

\section{$\mathrm{En}=\mathrm{En}-\mathrm{Etn}-\mathrm{Ern}-\mathrm{Emn}$}

Where, Emn is only for the high-energy moving nodes

Fig. 4 represents the scenario where all nodes are fixed and do not move for sensing. They sense the data from the position and send the same to base station. Whereas, in Fig. 5, all nodes move towards the sensing point. Fig. 6 tries to move selected nodes with high energy capacity and send the data to the base station but, other nodes will not send the data. The nodes are selected by pooling the energy levels of all nodes at base station, find the nodes with more energy than others in the network and make them to self-configure to move towards the sensing point.

\section{B. Proposed Algorithm}

Following are the steps of algorithm for running the simulation:

1) Deploy all the sensor nodes and the base station in the random positions in the sensing area.

2) Depending on one the following model, make the sensor nodes to act accordingly.

\section{a) Fixed nodes}

i. Initialize Total energy consumed by WSN Tnew(E) to zero

ii. Send messages from every node to base station

iii. Find the Total energy $\operatorname{Tnew}(\mathrm{E})$ at the end of simulation according to following relation:

$\mathrm{T}_{\text {new }}(\mathrm{E})=\mathrm{E}_{1}+\mathrm{E}_{2}+\ldots \ldots \ldots \ldots+\mathrm{E}_{\mathrm{n}}$

Where, E1,E2......En computed according to equation(1) after every transmission

b) All moving nodes

i. Initialize Total energy consumed by WSN Tnew(E) to zero,

ii. Send message from every node to base station,

iii. Move all the nodes near sensing area for better sensing,

iv. Find the Total energy $\operatorname{Tnew}(\mathrm{E})$ at the end of simulation according to following relation:

$\operatorname{Tnew}(\mathrm{E})=\mathrm{E} 1+\mathrm{E} 2+\ldots \ldots \ldots \ldots .+\mathrm{En}$

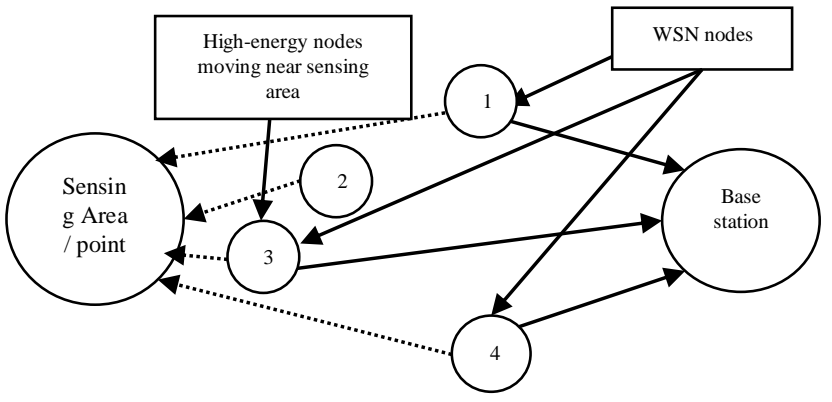

Fig. 6. Moving High Energy Nodes. 
Where, E1,E2.....En are computed according to equation(2) after every transmission.

\section{c) Move selected nodes}

i. Initialize Total energy consumed of WSN Tnew(E) to zero,

ii. Send messages from every node to base station,

iii. Find high and low energy nodes in the network after a message transmission and move them near sensing area for better sensing,

iv. Find the Total energy $\mathbf{T}_{\text {new }}(\mathbf{E})$ at the end of simulation according to following relation:

$\mathrm{T}_{\text {new }}(\mathrm{E})=\mathrm{E}_{1}+\mathrm{E}_{2}+\ldots \ldots \ldots \ldots+\mathrm{E}_{\mathrm{n}}$

Where, E1,E2.....En are computed according to equation(3) after every transmission

3) Record the events in the simulation before and after sending the messages for every model mentioned in previous step and analyze the events for different parameters of interest

\section{Assumptions}

1) A point sensing model is considered for simulations.

2) Number of nodes considered for simulation range from 10 to 50 sensors.

\section{Input Variables}

Input for experimentation is initial energy, number of nodes and other values. Table 1 indicates the input variables and the values acquired by them for the simulation.

TABLE I. INPUT VARIABLES

\begin{tabular}{|l|l|}
\hline Name of the variable & Range of values \\
\hline Number of WSN nodes & $10,20,30,40,50$ \\
\hline Initial Energy(in joules) & 10 \\
\hline Sending energy(Tx, in milijoules) & 1.8 \\
\hline Receiving energy(Rx, in milijoules) & 0.9 \\
\hline
\end{tabular}

E. Limitations

1) The proposed work has following limitations.

2) Number of nodes considered for study is 10 to 50 .

3) Sending and receiving energy is considered same for all nodes.

4) All QoS parameters are not considered for study.

\section{PARAMETERS FOR STUDY}

The work focuses on observing the change in the energy consumption with respect to the number of packets sent and because the movement of nodes in the sensing area. Hence, throughput, delay between the packets and energy consumption by the network are the parameters considered for study.

\section{Simulation ENVIRONMENT}

The work was simulated on Discrete Event network simulator Ns-2.34 tool, a known tool for conducting Wired, Wireless and Wireless Sensor Networks simulations. The WSN environment considered for the work has set of nodes initially randomly deployed out of which first node is made as base station and others as sensors. According to the type of deployment the sensors are made to send the sensed data (text message) to the base station. The self-configuring logic is implemented through the data collected from all the sensors in the base station.

\section{RESULTS AND ANALYSIS}

Simulation results were recorded for three different parameters of studies, namely throughput, end-to-end delay and energy consumed. In each of the tables from Table 2 to Table 4, difference column (fifth column) is calculated to compare the high energy moving kind of deployment with respect to other deployments with respect to the parameters of study. It is calculated as difference between the average of fixed and all moving deployments and high energy node movement deployment.

\section{A. Throughput}

Table 2 shows set of values of throughput observed for different number of nodes for simulation

Analysis: The number of extra packets sent from the nodes in the fixed and all moving kinds of deployment is found to be more than the high moving nodes in the network. As in each of the first two deployments, all nodes are sending the messages to base station. Where as in high-energy node deployment, the high-energy nodes get self-configured, move near the sensing area and send the data to base station. This is done just to ensure that high energy levels of nodes to be utilized instead of using the energy of all nodes in the network. Hence in high moving types of deployment less number of packets are sent from nodes as indicated by Table 2 and Fig. 7. It is observed that $10 \%$ to $15 \%$ decrease in the number of extra packets transmitted in high-energy node movement deployment compared to others with better sensing and better node energy utilization.

TABLE II. THROUGHPUT OF ALL PACKETS

\begin{tabular}{|l|l|l|l|l|}
\hline $\begin{array}{l}\text { Numb } \\
\text { er of } \\
\text { nodes }\end{array}$ & $\begin{array}{l}\text { High } \\
\text { moving } \\
\text { Through } \\
\text { put } \\
\text { (kbps) }\end{array}$ & $\begin{array}{l}\text { All } \\
\text { moving } \\
\text { Through } \\
\text { put } \\
\text { (kbps) }\end{array}$ & $\begin{array}{l}\text { Fixed } \\
\text { Through } \\
\text { put } \\
\text { (kbps) }\end{array}$ & $\begin{array}{l}\text { Difference = } \\
\text { (All+Fixed)/2 - High }\end{array}$ \\
\hline 10 & 82.09 & 83.93 & 87.14 & 3.445 \\
\hline 20 & 134.79 & 138.07 & 138.32 & 3.405 \\
\hline 30 & 190.12 & 201.48 & 219.85 & 20.545 \\
\hline 40 & 241.83 & 258.4 & 259.91 & 17.325 \\
\hline 50 & 305.8 & 321.51 & 351.36 & 30.635 \\
\hline
\end{tabular}




\section{Throughput comparison}

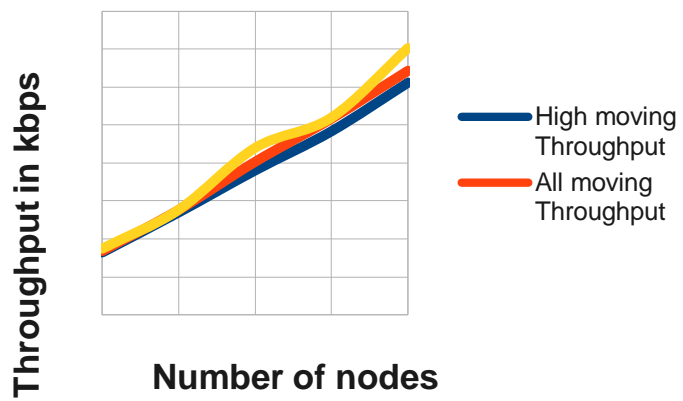

Fig. 7. Throughput of All Packets in Network.

\section{B. Delay}

Table 3 shows set of values of end to end delay between the packets delivered are observed for different number of nodes for simulation.

Analysis: As we can observe from the Table 3 and Fig. 8 the change in the delay from less number of nodes to more number of nodes in the network. Initially the delay is high in all deployments because all nodes are directly connected to the base station creating the load for base station to handle all the requests. The delay is almost same in all types of deployment but goes on decreasing as number of nodes increases and packet delivery becomes faster via more intermediate nodes. Finally, the delay becomes constant for all types of deployments.

TABLE III. END TO END DELAY

\begin{tabular}{|l|l|l|l|l|}
\hline $\begin{array}{l}\text { Numbe } \\
\text { r of } \\
\text { nodes }\end{array}$ & $\begin{array}{l}\text { High End } \\
\text { to End } \\
\text { delay } \\
\text { (in msec) }\end{array}$ & $\begin{array}{l}\text { All moving } \\
\text { End to } \\
\text { End delay } \\
\text { (in msec) }\end{array}$ & $\begin{array}{l}\text { Fixed End to } \\
\text { End delay } \\
\text { (in msec) }\end{array}$ & $\begin{array}{l}\text { Difference = (All } \\
\text { +Fixed)/2 - High }\end{array}$ \\
\hline 10 & 35.726 & 37.847 & 41.157 & 3.77645 \\
\hline 20 & 24.595 & 23.260 & 22.075 & -1.9269 \\
\hline 30 & 16.971 & 14.301 & 15.471 & -2.0854 \\
\hline 40 & 11.386 & 10.300 & 10.724 & -0.87365 \\
\hline 50 & 8.997 & 8.872 & 8.655 & -0.23403 \\
\hline
\end{tabular}

\section{End to End Delay comparison}

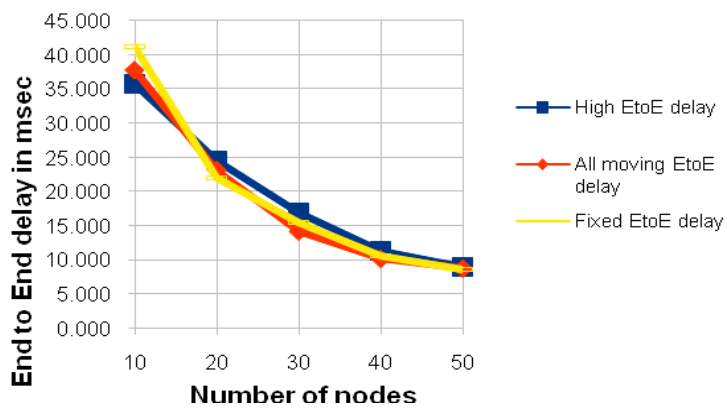

Fig. 8. End to End Delay of All Packets in Network.

\section{Energy}

Table 4 shows a set of values of End to end delay between the packet delivery observed for different number of nodes for simulation.

Analysis: As we can observe from Table 4 and Fig. 9 that the energy dissipation is less for high moving deployment than other deployments. This is because for the reason that all nodes are sending the data and in all moving nodes deployment all nodes are moving towards the sensing point. However, in case of high-energy nodes moving kind of deployment, only the selected nodes (the nodes with higher energy levels than the others at a instance of time) are allowed to move and transmit. This will help in best utilizing the available energy of all nodes and have better sensing effect as selected nodes are moving towards the sensing point. It is found from the difference column of Table 4 that there is an almost $6 \%$ better energy utilization in high-energy node movement deployment than other two deployment strategies.

Summary: In most of the HWSN application deployment of nodes play important role in achieving better performance without compromising the better sensing effect. Utilizing the high energy of nodes can reduce the number of access packets transmitted in the network. Even it can help utilizing the energy better than the other deployments without any concession on sensing effect. A better sensing effect means that if a node or nodes move towards the sensing point the quality of sensing definitely increases than the far away nodes.

TABLE IV. ENERGY CONSUMED BY THE NETWORK

\begin{tabular}{|l|l|l|l|l|}
\hline $\begin{array}{l}\text { Numbe } \\
\text { r of } \\
\text { nodes }\end{array}$ & $\begin{array}{l}\text { High } \\
\text { moving } \\
\text { Energy } \\
\text { (in mjoules) }\end{array}$ & $\begin{array}{l}\text { All moving } \\
\text { Energy } \\
\text { (in } \\
\text { mjoules) }\end{array}$ & $\begin{array}{l}\text { Fixed } \\
\text { Energy } \\
\text { (in } \\
\text { mjoules) }\end{array}$ & $\begin{array}{l}\text { Difference = (All } \\
\text { +Fixed)/2 - High }\end{array}$ \\
\hline 10 & 0.2709 & 0.3584 & 0.3048 & 0.060682 \\
\hline 20 & 0.3767 & 0.4085 & 0.3570 & 0.0060195 \\
\hline 30 & 0.3688 & 0.4132 & 0.3948 & 0.035184 \\
\hline 40 & 0.3265 & 0.3558 & 0.3226 & 0.0126765 \\
\hline 50 & 0.2773 & 0.3541 & 0.3253 & 0.0623898 \\
\hline
\end{tabular}

\section{Energy comparison}

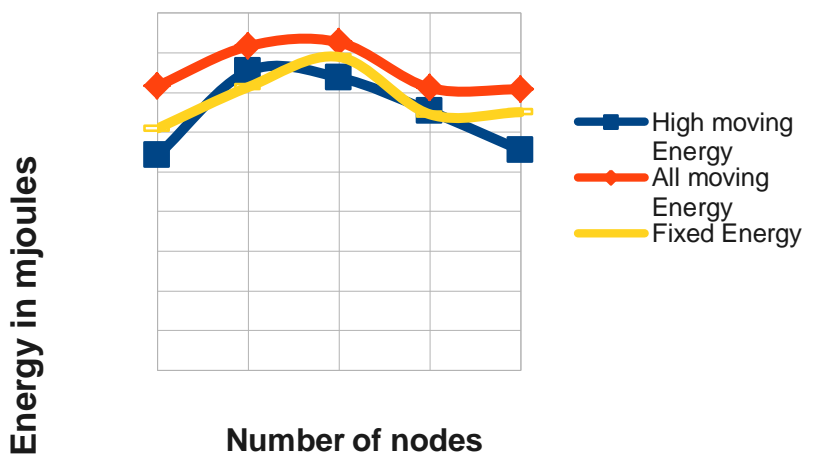

Fig. 9. Energy Consumed by All Nodes in Network. 


\section{CONCLUSION}

The main objective of most of WSN applications is the deployment of the nodes and better energy utilization schemes are key factors. Heterogeneity of nodes can play a vital role achieving this. Several deployment strategies are proposed for better sensing, less number of packet transmission, reduced packet delays and increased energy conservation. The work simulated three types of deployments like fixed node, all nodes moving and high-energy node moving deployments and found that high-energy moving nodes show $10 \%$ better throughput and $6 \%$ better energy utilization. This is possible as high energy nodes (energy heterogonous nodes) in dynamic deployment configure themselves to move towards sensing area for better sensing. Hence, it is observed from simulation results that dynamic deployment strategies with selfconfiguration logic of nodes can achieve better performance with respect to number of packets transmitted and energy utilization. In future, this work is planned, to be extended by computing the self-configuration logic using some Artificial intelligence or some machine learning technique. Especially, artificial neural network may be one of the best choices for it.

\section{REFERENCES}

[1] Haitao Zhang and Cuiping Liu, "A Review on Node Deployment of Wireless Sensor Network", IJCSI International Journal of Computer Science Issues, Vol. 9, Issue 6, No 3,pg. no. 378-383, November 2012.

[2] Nadjib Aitsaadi , Nadjib Achir , Khaled Boussetta , Guy Pujolle , "Artificial potential field approach in WSN deployment: Cost, QoM, connectivity, and lifetime constraints", pg. no. 55 (2011) 84-105, August 2010.

[3] Nusrat Mehajabin, Md. Abdur Razzaque , Mohammad Mehedi Hassan , Ahmad Almogren , Atif Alamri , “ Energy-sustainable relay node deployment in wireless sensor networks", ELSEVIER Journal of Computer Networks , pg. no. 104 (2016) 108-121 August 2010.

[4] Lili Wang, Chenfu Yi and Ye Li, "Energy Efficient Transmission Approach for WBAN Based on Threshold Distance", IEEE SENSORS JOURNAL, VOL. 15, NO. 9, pg. no. 5133 - 5141, SEPTEMBER 2015.

[5] Samay veer Singh, Energy efficient multilevel network model for heterogeneous WSNs" Engineering Science and Technology, an ELSEVIER International Journal.

[6] Abhaykumar L. Gupta and Narendra Shekokar, "A Novel Approach to Improve Network Lifetime in WSN by Energy Efficient Packet Optimization", 2nd IEEE International Conference on Engineering and
Technology (ICETECH), 17th \& 18th March 2016, Coimbatore, TN, India. DOI 978-1-4673-9916-6/16.

[7] Mohamed Ezz El Dien Abd El Kader, Aliaa A. A. Youssif, and Atef Zaki Ghalwash, "Energy Aware and Adaptive Cross-Layer Scheme for Video Transmission Over Wireless Sensor Networks", IEEE Sensors Journal, Vol.16, No. 21, November 1,2016.

[8] Eun-Jung Lee, Hea-Sook Park, Jun-Kyun Choi, Hong-Shik Park, Young-Min Kim , "Ant colony based self-adaptive energy saving routing for energy efficient Internet", ELSEVIER Journal of Computer Networks, Pg. no. 56 (2012) 2343-2354April 2012.

[9] Sinan Toklu, O. Ayhan Erdem, "BSC-MAC: Energy efficiency in wireless sensor networks with base station control", ELSEVIER Journal of Computer Networks, . Pg. no. 59 (2014) 91-100, December 2013.

[10] NaumanAhad,JunaidQadir,NasirAhsan, "Neural networks in wireless networks : Techniques, applications and guidelines", Journal of Network and Computer Applications, April 2016, pg. no. 1-27.

[11] Hui Wang, H. Eduardo Roman , Liyong Yuan , Yongfeng Huang, Rongli Wang, "Connectivity, coverage and power consumption in large-scale wireless sensor networks", ELSEVIER Journal of Computer Networks, 2014, pg. no. 52 2419-2431.

[12] Wei-Chieh Ke , Bing-Hong Liu, Ming-Jer Tsai, "The critical-squaregrid coverage problem in wireless sensor networks is NP-Complete", ELSEVIER Journal of Computer Networks,pg. no. 55 (2011) 22092220, March 2011.

[13] Durga Pavan Nudurupati, Rajat Kumar Singh, "Enhancing Coverage Ratio using Mobility in Heterogeneous Wireless Sensor Network", ELSEVIER Proceedings Technology 10 ( 2013 ) 538 - 545 of International Conference on Computational Intelligence: Modeling Techniques and Applications", (CIMTA) 2013.

[14] Gupta, S., Parveen, N. , “Optimum Node Deployment Strategy for Heterogeneous Wireless Sensor Network by Estimating Network Lifetime" published in Emerging Trends in Engineering and Technology (ICETET), 2009 2nd International Conference on Date 16-18 Dec. 2009.

[15] Dilip Kumar, e.t.a.1, "EEHC: Energy efficient heterogeneous clustered scheme for wireless sensor networks", published in Computer Communications journal, published by Elsevier, pg. no.32 (2009) 662667, 2008.

[16] Sabrina Sicari, Alessandra Rizzardi, Luigi Alfredo Grieco, and Alberto Coen-Porisini, "Performance Comparison of Reputation Assessment Techniques Based on Self-Organizing Maps in Wireless Sensor Networks", Hindawi Wireless Communications and Mobile Computing Volume 2017.

[17] Nusrat Mehajabin , Md. Abdur Razzaque , Mohammad Mehedi Hassan , Ahmad Almogren, Atif Alamri, “ Energy-sustainable relay node deployment in wireless sensor networks", ELSEVIER Journal of Computer Networks , pg. no. 104 (2016) 108-121. 